\title{
Concentração espacial da indústria de São Paulo: evidências sobre o papel da disponibilidade de gás natural
}

EDGAR ANTONIO PERLOTTI, I

EDMILSON MOUTINHO DOS SANTOS II

e Hirdan Katarina de MEDEIROS COSTA ${ }^{I I I}$

\section{Introdução}

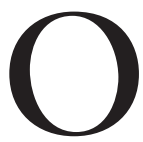

GÁs NATURAL é um dos energéticos mais importantes da matriz brasileira, ocupando o quinto lugar entre os mais consumidos e respondendo por 7,6\% do consumo final de energia em 2011 (Empresa de Pesquisa Energética, 2012a). Além de sua representatividade na matriz energética, o gás natural foi o energético que mais ganhou participação ao longo da década de 2000.

Particularmente, o setor industrial foi responsável por esse avanço, respondendo por $11,3 \%$ do consumo final de energia. $O$ volume consumido na atividade industrial é cerca de metade do total de gás natural consumido no país. No final da década de 2000, a Região Sudeste do Brasil concentrava a quase totalidade do consumo, embora se note um aumento da importância do Nordeste como região consumidora, ao mesmo tempo que a atividade econômica da região se acelera.

$\mathrm{O}$ avanço do energético na indústria se deveu ao aumento da oferta e da melhora do preço relativo do gás natural em relação ao seu principal substituto, o óleo combustível. Como resposta à crise de 2008, houve um excedente de oferta de gás natural no mundo, resultando em redução de preços. As expectativas de aumento de oferta, por conta do desenvolvimento de gás natural em campos não convencionais, principalmente nos Estados Unidos, também colaboraram para redução do preço. Adicionalmente, há um aumento da preferência pelo gás natural em processos industriais que exigem elevado grau de pureza do produto final, como a fabricação de cerâmica e vidro, por conta da elevação do valor adicionado do produto e seu melhor posicionamento no mercado exportador (Empresa de Pesquisa Energética, 2012b).

Consequentemente, há uma grande preocupação em se discutir e planejar o segmento de gás natural no longo prazo. Em 2012, observou-se grande vo- 
lume de apresentações, discussões, seminários e desenvolvimento de pesquisas tendo como tema o futuro do gás natural. Destaque pode ser dado aos esforços do Projeto +Gás Brasil, capitaneado pela Associação Brasileira de Grandes Consumidores Industriais de Energia e Consumidores Livres (Abrace). O projeto propõe uma discussão sobre o futuro do gás natural na matriz energética brasileira, fazendo diagnósticos de mercado e apontando os potenciais de investimento e impactos na economia nacional (+Gás Brasil, 2012).

Nesse sentido, torna-se fundamental que diversas questões relevantes sejam colocadas em discussão. Este artigo se propõe a desenvolver uma análise sobre o papel da disponibilidade de gás natural como fator relevante para a instalação e funcionamento de indústrias, ou setores industriais, cujo produto final obtenha adição de valor pela utilização do energético, utilizando o estado de São Paulo como estudo de caso.

Em última análise, busca-se reconhecer que a disponibilidade de energia, ao lado de outros elementos, particularmente a disponibilidade de infraestrutura eficiente, é um fator de atratividade da produção industrial e, por extensão, de desenvolvimento econômico regional. Cabe ressaltar que a produção de literatura sobre concentração geográfica da atividade econômica é bastante extensa no Brasil, porém os vínculos entre energia e localização industrial ainda são pouco explorados nesta literatura.

Para tanto, a seção seguinte apresenta a conjuntura do gás natural no Brasil, principalmente com relação à utilização do energético na indústria e às condições de transporte do gás. A terceira seção apresenta o arcabouço teórico e empírico da concentração espacial industrial.

$\mathrm{Na}$ quarta seção é detalhada a metodologia utilizada para a seção empírica deste trabalho e, na quinta, são apresentados os resultados obtidos do estudo de caso: estado de São Paulo. Finalmente, a última seção traz as considerações finais do trabalho.

\section{Panorama da indústria do gás natural no Brasil}

Embora seja uma indústria muito antiga, a concorrência com outros energéticos e os elevados custos de transporte retardaram o uso extensivo de gás natural no mundo. A evolução da indústria do gás natural esteve intimamente ligada à indústria do petróleo, uma vez que o preço do óleo determinava a viabilidade de exploração do gás em uma dada área - o gás era explorado, usualmente, quando associado à produção de petróleo e em locais onde o mercado consumidor estivesse próximo (Marques, 2009).

No período posterior aos choques do petróleo, na década de 1970, o elevado preço do gás viabilizou investimentos de grande porte em infraestrutura de transporte. O descolamento desse mercado em relação ao mercado de petróleo levou ao crescimento do gás natural na matriz energética mundial

O uso industrial do gás natural está vinculado diretamente aos preços relativos aos seus substitutos, ao custo de conservação das instalações, à impor- 
tância dos ganhos de eficiência na competitividade do produto final, ao grau de exigências ambientais sobre o processo produtivo e à disponibilidade de linhas de financiamento (Marques, 2009).

A aplicação de gás natural nos processos industriais se destaca pela eficiência de sua utilização em fornos a altas temperaturas, pela pequena emissão de poluentes quando do uso de vapor gerado por caldeiras para usos na indústria química, farmacêutica, de papel e celulose e de alimentos e bebidas, pelo seu potencial na substituição da eletrotermia (aquecimento ou geração de vapor por meio de eletricidade), na operação de calor direto para secagem e fabricação de cimentos e refratários. Também se destaca por seu potencial como matéria-prima e redutor siderúrgico. De forma geral, o uso de gás natural leva a produtos de melhor qualidade, eliminando resíduos de combustão incompleta ou metálicos e de óxidos de enxofre (Moutinho dos Santos, 2002).

Alguns segmentos da indústria de cerâmica são exemplos clássicos de aumento de competitividade em razão da utilização do gás natural. O segmento de cerâmica de revestimento aumentou a qualidade e o valor agregado do produto final com a utilização de gás natural no seu processo de queima e secagem (Pacheco, 1999).

O uso do gás natural no Brasil tem seu início na década de 1940, com as descobertas de óleo e gás no Recôncavo Baiano. A produção era praticamente toda destinada às indústrias da região e, mais tarde, ao polo petroquímico de Camaçari. As descobertas na Bacia de Campos, a partir de 1980, levaram a um aumento significativo das reservas provadas no país (Faria, 2010).

A indústria de gás natural foi marcada, na década de 1990, pela construção do Gasoduto Bolívia-Brasil, o Gasbol. Em 1999, o Gasbol entrou em operação, levando a um aumento significativo da oferta de gás natural disponível. Posterior à crise de falta de energia elétrica, em 2001, houve um movimento do governo federal no sentido de se incentivar a construção de usinas termelétricas movidas a gás natural, com destaque para o Programa Prioritário de Termelétricas (PPT) e o Plano de Massificação do Uso de Gás Natural, da Petrobras. Com o expressivo crescimento da demanda nesse período, a oferta de gás natural no país passou a ser insuficiente para atender as indústrias e o setor energético (Faria, 2010).

O cenário desfavorável colocou em dúvida a capacidade de sustentabilidade da indústria de gás natural. Porém, a partir de 2009, dois movimentos levaram a um novo momento positivo para o setor. De um lado, a crise econômica levou à redução de consumo, aumentando a disponibilidade de gás e, por consequência, à diminuição dos preços. Mais importante, houve um aumento na oferta de gás, advindo do desenvolvimento dos campos não convencionais.

Esses campos, localizados principalmente na América do Norte, se caracterizam pela elevada complexidade em sua exploração. Um grande conjunto de elementos, que extrapolam o alcance deste trabalho, explica o momento oportuno para exploração destes campos. No Brasil, em particular, estima-se que 
existam reservas não convencionais onshore com volumes superiores às grandes descobertas do Pré-Sal (+Gás Brasil, 2012).

O gás natural brasileiro, atualmente, é processado nas seguintes unidades (pontos de oferta): Urucu no Amazonas; Lubnor no Ceará; Guamaré no Rio Grande do Norte; Pilar em Alagoas; Carmópolis e Atalaia em Sergipe; Catu, Candeias e São Francisco do Conde na Bahia; Lagoa Parda, Cacimbas e Ubu no Espírito Santo; Cabiúnas e Reduc no Rio de Janeiro; Merluza e Caraguatatuba em São Paulo. Adicionalmente estão disponíveis ou serão disponibilizados em breve: Corumbá, Mato Grosso do Sul, para o gás importado pelo Gasbol; Terminais de regaseificação de Gás Natural Liquefeito (GNL) em Pecém-CE, Baía de Guanabara-RJ e Bahia; São Antonio dos Lopes, na bacia do Parnaíba (MA); e os futuros terminais de Parecis (MT), Regap (MG) e Refap (RS).

O setor industrial é o mais importante consumidor de gás no país. Entre os setores industriais, destacam-se a química (onde o gás natural é utilizado também como matéria prima), a cerâmica e a produção de ferro-gusa e aços. Na Tabela $\mathrm{l}$ foi dado destaque à indústria têxtil por duas razões: houve crescimento expressivo do consumo do energético no segmento ao longo da década (embora não superior ao de outros setores, de forma que não houve ganho relativo de participação), mesmo com a expressiva redução na atividade econômica deste setor, e, principalmente, o gás natural ocupou espaço significativo na matriz energética do segmento (quase um terço), deslocando o uso de outros energéticos, como a eletricidade.

Tabela 1 - Participação do gás natural na matriz energética dos segmentos industriais $(\%)$

\begin{tabular}{|c|c|c|c|c|c|c|c|c|c|c|}
\hline & 2002 & 2003 & 2004 & 2005 & 2006 & 2007 & 2008 & 2009 & 2010 & 2011 \\
\hline Industrial & $9 \%$ & $9 \%$ & $9 \%$ & $10 \%$ & $10 \%$ & $10 \%$ & $10 \%$ & $10 \%$ & $11 \%$ & $11 \%$ \\
\hline Cimento & $1 \%$ & $1 \%$ & $1 \%$ & $1 \%$ & $1 \%$ & $1 \%$ & $1 \%$ & $1 \%$ & $1 \%$ & $1 \%$ \\
\hline Ferro-Gusa e Aços & $6 \%$ & $5 \%$ & $5 \%$ & $6 \%$ & $7 \%$ & $7 \%$ & $7 \%$ & $5 \%$ & $5 \%$ & $6 \%$ \\
\hline Ferro-Ligas & $0 \%$ & $0 \%$ & $0 \%$ & $0 \%$ & $0 \%$ & $2 \%$ & $0 \%$ & $0 \%$ & $0 \%$ & $0 \%$ \\
\hline Mineração e Pelotização & $8 \%$ & $8 \%$ & $9 \%$ & $9 \%$ & $9 \%$ & $7 \%$ & $13 \%$ & $8 \%$ & $20 \%$ & $21 \%$ \\
\hline $\begin{array}{l}\text { Não Ferrosos e Outros } \\
\text { da Metalurgia }\end{array}$ & $6 \%$ & $7 \%$ & $9 \%$ & $9 \%$ & $9 \%$ & $11 \%$ & $11 \%$ & $8 \%$ & $11 \%$ & $11 \%$ \\
\hline Química & $25 \%$ & $25 \%$ & $29 \%$ & $30 \%$ & $30 \%$ & $29 \%$ & $32 \%$ & $31 \%$ & $32 \%$ & $33 \%$ \\
\hline Alimentos e Bebidas & $3 \%$ & $3 \%$ & $3 \%$ & $3 \%$ & $3 \%$ & $3 \%$ & $3 \%$ & $3 \%$ & $3 \%$ & $3 \%$ \\
\hline Têxtil & $21 \%$ & $24 \%$ & $25 \%$ & $28 \%$ & $28 \%$ & $29 \%$ & $27 \%$ & $26 \%$ & $27 \%$ & $27 \%$ \\
\hline Papel e Celulose & $6 \%$ & $6 \%$ & $6 \%$ & $7 \%$ & $7 \%$ & $7 \%$ & $6 \%$ & $5 \%$ & $7 \%$ & $7 \%$ \\
\hline Cerâmica & $23 \%$ & $25 \%$ & $24 \%$ & $26 \%$ & $26 \%$ & $25 \%$ & $24 \%$ & $24 \%$ & $26 \%$ & $28 \%$ \\
\hline Outros & $15 \%$ & $16 \%$ & $17 \%$ & $17 \%$ & $17 \%$ & $18 \%$ & $20 \%$ & $20 \%$ & $26 \%$ & $26 \%$ \\
\hline
\end{tabular}

Fonte: Empresa de Pesquisa Energética, 2012a. 
O gás natural possui potencial para utilização em uma diversidade de setores industriais como substituto eficiente em vários processos. A Tabela 2 mostra exemplos de setores e energéticos que podem ser eficientemente deslocados pela adoção de gás natural. Como destaca Moutinho dos Santos (2002, p.101), “a versatilidade é uma das grandes vantagens do gás natural. [...] Porém, ao mesmo tempo, não existe uma aplicação para o gás natural na qual ele seja indispensável e para o qual não haja concorrentes".

Tabela 2 - Energéticos deslocados na matriz energética pelo utilização de Gás Natural

\begin{tabular}{ll}
\hline Uso no Setor Industrial & Substituição de Energéticos \\
\hline Fabricação de Aço & Óleo combustível, gás de alto forno, eletricidade \\
\hline Processamento de Aço & Coque \\
Metalurgia (diversos) & Óleo combustível, gás de alto forno, eletricidade \\
Minerais Não Metálicos & Eletricidade, óleo combustível, GLP \\
Alimentos e Bebidas & Óleo combustível, coque, carvão pulverizado, biomassa \\
Caldeiras & GLP, diesel, eletricidade \\
Equipamentos de torrefação & Eletricidade, óleo combustível, GLP \\
Têxteis & GLP, óleo combustível, lenha \\
Papel e Celulose & Óleo combustível, lenha, carvão \\
Química / Petroquímica & Óleo combustível, nafta, eletricidade \\
\hline
\end{tabular}

Fonte: Moutinho dos Santos (2002).

\section{Do transporte de gás natural}

A cadeia produtiva do gás natural é constituída por três principais componentes: upstream, midstream e downstream. A etapa upstream é formada pela exploração, explotação e processamento. $\mathrm{O}$ midstream corresponde às etapas de armazenagem, transporte de grandes volumes para abastecer consumidores industriais de grande porte e geração termelétrica. Por fim, a etapa de downstream é aquela em que ocorrem as negociações e distribuição em baixa pressão para atendimento de consumidores residenciais, comerciais e pequenas indústrias (Rigolin, 2007).

A etapa inicial, o upstream, emprega as mesmas tecnologias de exploração e produção de petróleo, embora o gás natural possa estar associado ou não ao óleo. Os poços podem estar localizados em terra, quando são chamados de onshore, ou no mar, offshore. Parte do gás produzido nos campos, depois de ser 
separado da água e outros hidrocarbonetos em estado líquido e descontaminado do eventual enxofre, é utilizada para geração de eletricidade e vapor e, também, é reinjetada nos campos, aumentando a recuperação de petróleo do reservatório. O gás restante ou é enviado para processamento ou é queimado (flares).

$\mathrm{Na}$ sequência da cadeia, o gás natural segue para Unidades de Processamento de Gás Natural (UPGN), onde é beneficiado e fracionado. O metano é o principal hidrocarboneto presente, com participação de até $95 \%$ do conteúdo total do gás (Rigolin, 2007).

As principais formas de transporte do gás natural são: gasodutos, gás natural liquefeito (GNL) e gás natural comprimido (GNC). Os gasodutos de alta pressão, a forma mais comum de transporte, exigem elevados volumes de investimento e apresentam características de monopólio natural. Trata-se de uma típica indústria de rede, onde a integração espacial é rígida.

Os gasodutos principais costumam ter entre 16 e 42 polegadas de diâmetro, enquanto suas ramificações possuem diâmetros entre 6 e 16 polegadas. Esses gasodutos são produzidos com segmentos de aço unidos através de soldas especiais e revestidos com camadas protetoras (usualmente resina de epóxi anticorrosivo).

A construção de um gasoduto de transporte de gás natural é complexa e exige meses, ou até anos, de estudos e planejamento. Depois de determinado o traçado, considerando as necessidades energéticas presentes e potenciais de uma dada região, é feita uma limpeza da linha, incluindo cortes de árvores, superação de barreiras e obstáculos para a entrada de maquinário. Os dutos são instalados em uma vala com profundidade entre 1 e 4 metros e cobertos com uma camada de terra. Quando da instalação de dutos em regiões alagadas, a linha é protegida por caixas de concreto (Rigolin, 2007).

O transporte feito através da liquefação do gás natural, GNL, exige o resfriamento do energético e é uma alternativa para regiões onde não exista estrutura de gasodutos disponível. O GNL precisa ser transportado por navios metaneiros e regaseificado em terminais de recepção. Outra forma alternativa de transporte é através da compressão do gás, GNC, que é transportado em cilindros especiais.

A malha de rede nacional de gasodutos possui, em 2012, um total de $9.489,2 \mathrm{~km}$ em todas as regiões brasileiras. A malha integrada é operada por três operadores: a Transportadora Sulbrasileira de Gás (TSB), que opera no Rio Grande do Sul o gasoduto Uruguaiana-Porto Alegre; a Transportadora Brasileira Gasoduto Bolívia-Brasil (TBG) opera o Gasbol; a Transpetro que opera o restante da malha integrada (Empresa de Pesquisa Energética, 2012b).

A maior parte da malha de gasodutos brasileira foi construída ao longo das décadas de 1980 e 1990, dentro do âmbito da política nacional de incentivo ao consumo de gás natural. Com a construção do Gasoduto Bolívia-Brasil, no começo da década de 2000, houve uma forte ampliação da malha. Mais recente- 
mente, o Projeto Malhas, proposto pela Petrobras, tem como objetivo expandir o sistema de gasodutos do Nordeste e do Sudeste, garantindo o suprimento de gás natural para usinas, por até 20 anos (Agência Nacional do Petróleo, 2011).

O Gasoduto Bolívia-Brasil (Gasbol) possui grande importância econômica e política para a indústria do gás natural brasileira. $\mathrm{O}$ traçado do gasoduto possui $3.150 \mathrm{~km}$ de extensão, dos quais $2.593 \mathrm{~km}$ estão em território nacional. $\mathrm{O}$ Gasbol tem início na cidade boliviana de Santa Cruz de La Sierra e atravessa os estados de Mato Grosso do Sul, São Paulo, Paraná, Santa Catarina e Rio Grande do Sul, terminando na cidade gaúcha de Canoas.

O Gasbol possui uma capacidade de transporte de até 30 milhões de metros cúbicos de gás natural por dia e é formado por dois trechos: o Norte, que liga Corumbá (MS) a Guararema (SP), e o Sul, que liga Paulínia (SP) a Canoas (RS).

Por fim, ainda com relação à cadeia do gás natural, há o sistema de distribuição, que compreende a movimentação de gás natural desde os pontos de entrega do sistema de transporte (city gates) até o consumidor final. A responsabilidade sobre a distribuição é de concessionárias de serviço público, sujeitas à regulação estadual, com contratos de exclusividade nas áreas de concessão. $\mathrm{O}$ modelo usual na formação das distribuidoras de gás natural no Brasil é a constituição de três sócios: governo estadual, ente privado e a Petrobras.

O preço do gás natural vendido às distribuidoras de gás é formado por duas parcelas: o custo da commodity, utilizado para remunerar o produtor, e o custo de transporte.

\section{Da concentração industrial}

A fundamentação econômica sobre localização e concentração da atividade econômica tem início nos anos 1920, com os trabalhos seminais de Marshall (1920) e Weber (1929), e este último classificou os fatores que determinam a localização das firmas como regionais (relacionados à dotação geográfica) e locais (independentes da geografia, como qualidade da infraestrutura urbana, custo da terra, economias de escala). A linha de pesquisa conhecida como Nova Geografia Econômica refere-se a esses fatores como forças centrípetas, quando estimulam a concentração espacial, e forças centrífugas, quando promovem a desconcentração (Krugman, 1991).

Diversas variáveis são apontadas na literatura para explicar a decisão de localização de uma firma. Distintos modelos também são construídos para explicar a relação entre estas variáveis e o processo de escolha do local de produção e comercialização do produto (Tirole, 1988; Abdel-Rahman; Fugita, 1990; Fujita; Thisse, 2001; McCann, 2002; Edwards, 2006). Dentre essas diversas variáveis que podem ser utilizadas para explicar o processo de espacialização da indústria, a disponibilidade e o preço da energia foram pouco explorados na literatura. A maior parte dos trabalhos aborda o impacto dos preços de energia elétrica sobre a localização da indústria (Nijkamp; Perrels, 1988). 
Alternativamente trabalha-se com aberturas setoriais, identificando aqueles setores em que a energia representa um custo significativo, e, portanto, localizar-se em regiões com abundância de oferta (ou, similarmente, preços reduzidos pela concorrência) gera ganhos de competitividade (Worrel et al., 1997).

A literatura nacional dentro desse tema é escassa, mas existem indicativos de que preço e disponibilidade de energia podem representar importante fator de localização de indústrias energo-intensivas (Santos, 2012). Particularmente, a disponibilidade de gás natural no Brasil, por conta das características de transporte e distribuição, é um potencial candidato à geração de padrões de localização da indústria nacional (Piquet; Miranda, 2009).

\section{Fundamentos teóricos da economia de localização}

Estudos sobre localização e concentração espacial da atividade econômica são desenvolvidos deste o início do século, como já mencionado. Os trabalhos na área de localização industrial apontam para existência de forças que estimulam a concentração ou a desconcentração industrial, com o intuito de explicar a dinâmica espacial da indústria. A chamada Ciência Regional fornece o arcabouço conceitual para os fatores locacionais da atividade econômica (Resende; Wyllie, 2005). Particularmente, em economias de grande porte e heterogêneas, como a brasileira, há um especial interesse no desenvolvimento de análises que orientem políticas de desenvolvimento regional.

Os primeiros trabalhos desenvolvidos dentro dessa área tratavam as chamadas externalidades marshallianas como fatores de aglomeração industrial. As externalidades também foram fundamentais para a teorização em torno das aglomerações urbanas e das diversas linhas de pesquisa da Geografia Econômica. Nos anos 1990, o foco das pesquisas foi a microfundamentação dos argumentos para explicar a aglomeração das atividades econômicas (Silva; Silveira Neto, 2009).

Esses modelos da década de 1990 analisaram as razões pelas quais as firmas se localizam próximas aos compradores e vendedores (Krugman, 1991; Tirole, 1988).

Fujita e Thisse (2001) demonstram que a proximidade com os insumos de produção favorece a aglomeração, enquanto a produtividade marginal decrescente e os efeitos de congestão podem conduzir à dispersão das atividades econômicas.

Com relação aos determinantes de aglomeração da atividade econômica, a literatura aponta para dois tipos principais de externalidades. As economias de localização se referem aos determinantes que beneficiam firmas da mesma indústria, onde os fatores explicativos são específicos do setor de atividade. As economias de urbanização se referem aos determinantes que afetam indistintamente diferentes indústrias (Resende; Wyllie, 2005). 


\section{Literatura empirica no Brasil}

A literatura brasileira sobre os condicionantes da localização industrial se divide em duas vertentes básicas. Há um grupo cujo interesse está na análise histórica da concentração e trata como condicionantes da concentração industrial elementos estruturais das economias locais (Cano, 1998). Outra vertente, na qual estão incluídos os trabalhos mais recentes, se dedica a uma análise mais qualitativa do processo de concentração industrial e empreende esforços no sentido de quantificar a análise.

De forma geral, os estudos empíricos aplicados ao caso brasileiro concluem que existem evidências favoráveis aos fatores que condicionam a concentração industrial. Destacam-se os custos de transporte de bens, as economias externas, como o transbordamento do conhecimento e da informação e os ganhos de localização decorrentes de ligações entre oferta e demanda.

Usualmente, para se mensurar a concentração geográfica, utiliza-se um índice de dispersão, comumente a variância de alguma variável de atividade econômica. Diversos autores apontam para utilização dos números de emprego formal, disponíveis na Relação Anual de Informações Sociais (Rais) do Ministério do Trabalho e Emprego, como proxy do nível de atividade industrial.

Diniz (1995), usando os dados da Rais, além de dados do Censo Industrial do Instituto Brasileiro de Geografia e Estatística (IBGE), calcula medidas de concentração baseadas no valor de transformação industrial e no nível de emprego. O autor mostrou o processo de concentração da atividade econômica na Região Sudeste, mesmo com desconcentração nas grandes cidades.

Pacheco (1999) utiliza medidas semelhantes às empregadas por Diniz (1995) e mostra uma desconcentração da atividade econômica no país entre 1986 e 1996. O autor também conclui que os padrões setoriais de concentração são bastante distintos, evidenciando a necessidade de desenvolver a análise com a maior desagregação setorial possível.

Suzigan et al. (2001) utilizam as informações de emprego da Rais e calculam o processo de concentração em microrregiões do estado de São Paulo. O estudo verifica uma maior dinamização de regiões onde a indústria se especializou. Também ressalta as distinções entre setores de atividade, reforçando a necessidade de se tomar cuidados adicionais na formulação de políticas industriais.

Oliveira (2004) destaca a correlação positiva entre variáveis de nível educacional e crescimento da atividade econômica no Nordeste. Chagas e Toneto Júnior (2003) encontram correlação positiva entre especialização econômica e crescimento. Domingues (2005) evidencia o papel negativo dos custos de transporte, e Silveira Neto (2005) destaca que as economias de escala foram responsáveis pelas aglomerações industriais no final da década de 1990. Em comum, os diversos autores mostram que conclusões importantes surgem da análise das informações espacialmente desagregadas. 
Piquet e Miranda (2009) ressaltam o fato de que a grande concentração dos gasodutos de transporte de gás natural pode levar à concentração industrial. De fato, os ganhos decorrentes da redução de custo com o transporte do gás e os ganhos de valor de produção derivados da utilização do energético como insumo podem ser candidatos a fatores de aglomeração, de acordo com as teorias correntes de localização industrial.

\section{Metodologia}

Ellison e Glaeser (1994) se concentraram na tarefa de colaborar com a nascente linha de pesquisa da Nova Geografia Econômica, através da definição de índices de concentração geográfica ou espacial da atividade econômica. Ao longo de seu trabalho, os autores reforçam que sua intenção é utilizar o termo "concentrada" para definir o conjunto de indústrias que apresentem níveis de concentração superiores àqueles que são observados para a média da indústria total.

Assim sendo, mesmo que o empresário escolha a localização de sua unidade fabril jogando um dardo no mapa no país seria possível observar algum processo de concentração. Desse modo, assim como os autores, este trabalho irá definir como concentradas apenas aquelas indústrias cujo padrão de concentração é superior ao observado na média da indústria total.

Cabe ressaltar que o termo indústria irá definir, exceto que explícito o contrário, um segmento industrial qualquer - cerâmica, química, alimentos e bebidas, por exemplo. Ademais, a aleatoriedade na variável localização industrial estará sempre associada, informalmente, à ideia de lançamento de dardos em um mapa. Ou seja, quando a escolha do local de instalação de uma firma é aleatória, o empresário não toma sua decisão baseada em nenhum elemento explícito. Essa imagem é amplamente utilizada em trabalhos que tratam de localização espacial da atividade econômica.

Duas propriedades importantes devem ser observadas na construção de um índice de concentração geográfica ou espacial: o índice deve medir algo que seja do interesse do analista e permitir que sejam feitas comparações entre indústrias. As premissas básicas por trás do modelo de Ellison e Glaeser (1994), aqui chamado de Modelo EG, consideram que a escolha de localização é dada pela presença de outras empresas do setor (aglomeração gera externalidades positivas) e de vantagens naturais, como a proximidade do mercado de insumos. No caso deste trabalho, há um particular interesse em relação à proximidade do sistema de transporte de gás natural.

Usualmente os dados utilizados para medir concentração industrial se referem ao número de empregados por segmento industrial. Uma região (país, por exemplo) é dividida em M sub-regiões. Cada uma destas sub-regiões possui um percentual do emprego total, definidos como $x_{1}, x_{2}, x_{3}, \cdots, x_{M}$. Para cada segmento industrial é possível calcular a participação do emprego em cada sub-região de forma análoga, obtendo $s_{1}, s_{2}, s_{3}, \cdots, s_{M}$. 
Uma medida simples de aglomeração é dada por:

$$
g=\sum_{i=1}^{M}\left(s_{i}-x_{i}\right)^{2}
$$

O número dado por $g$ mostra o quanto a participação de empregados em um dado setor se distancia da participação "padrão" de empregados na economia. Ou seja, trata-se de uma medida de variância do emprego na região para um dado setor.

O índice é calculado para cada atividade industrial e permite uma comparação simples entre as atividades. Individualmente, os valores não possuem significado claro. Os valores são úteis quando utilizados para comparar setores ou um mesmo setor ao longo do tempo. Setores que possuem índices mais elevados são mais concentrados que os demais, uma vez que sua distribuição geográfica é bastante distinta da média dos setores.

O termo entre parênteses é elevado ao quadrado de forma que o resultado seja positivo em todos os casos e que haja uma penalização de setores com variâncias mais elevadas.

Uma questão importante em relação ao índice $g$ diz respeito a indústrias que possuem poucas empresas na região em análise. Suponha um segmento industrial, como exemplo a fabricação de equipamentos médico-hospitalares de alta tecnologia, cujas características intrínsecas não permitem a instalação de um grande número de unidades ao longo de uma região (demanda muito especializada e, portanto, reduzida e exigência de mão obra com elevada qualificação). Pode se imaginar que serão instaladas uma ou duas empresas no território. Provavelmente, essas poucas unidades industriais não possuirão um número elevado de empregados. Porém, o índice irá considerar que essa indústria apresenta concentração espacial, uma vez que a média de emprego da indústria em uma dada sub-região será elevada (pelo simples fato de que não haverá outros empregos desta indústria em outras sub-regiões).

A solução para esse problema está na consideração de um índice de concentração econômica da atividade industrial. Por exemplo, pode-se lançar mão de uma adaptação do índice de Herfindahl, que quantifica a concentração de emprego dentro da indústria (Hirschman, 1964). Consideremos uma indústria formada por $N$ plantas, cada uma apresentando participação no emprego da atividade $z_{1}, z_{2}, z_{3}, \cdots, z_{N}$. O índice de Herfindahl é dado pela seguinte equação:

$$
H=\sum_{j=1}^{N} z_{j}^{2}
$$

Suponha que existam em uma dada indústria seis grandes empresas, onde cada uma detém $15 \%$ do emprego do segmento. Outras dez pequenas empresas possuem 1\% do emprego cada. Assim, seu índice de Herfindahl é da ordem de 0,136 . 
Para outro segmento, suponhamos que exista apenas uma empresa que detém $90 \%$ do emprego industrial, além de outras dez pequenas empresas que dividem igualmente o restante dos empregos. O índice de Herfindahl para essa indústria será da ordem de 0,643 . Nesse caso, é possível afirmar que esse segmento é mais concentrado, do ponto de vista econômico, que o anterior. Porém, essa concentração não diz nada a respeito da concentração geográfica da atividade.

Como as questões relativas à concentração econômica não estão dentro do escopo da análise de concentração geográfica, pode-se utilizar o índice de Herfindahl como variável de ajuste dos índices de concentração, eliminando resultados indesejáveis. Essa é a solução metodológica adotada pelo Modelo EG.

A base de dados utilizada para construção dos índices do Modelo EG, seguindo as sugestões presentes na maior parte da literatura empírica nacional, foram os números de empregados da Relação Anual de Informações Sociais (Rais), do Ministério do Trabalho e Emprego (MTE).

\section{Estudo de caso: a concentração espacial da indústria de São Paulo e indústria do GN}

O estado de São Paulo é a maior economia do país, concentrando um terço do PIB nacional. Possui também o segundo maior PIB per capita e o terceiro maior Índice de Desenvolvimento Humano (IDH). A indústria paulista é responsável por quase metade do PIB estadual e conta com diversos polos: indústria automobilística na Região Metropolitana de São Paulo, as indústrias de alta tecnologia nas Regiões Centrais (São Carlos e Araraquara), Vale do Paraíba e Região Metropolitana de Campinas e a agroindústria na Mesorregião de Piracicaba (IBGE, 2012).

Para o estado de São Paulo procedeu-se, inicialmente, à determinação do nível de concentração econômica das indústrias, calculando-se o Índice de Herfindahl, apresentado na Tabela 3. Ao contrário do que é observado no nível nacional, há um aumento significativo de concentração econômica para um conjunto significativo de setores, principalmente a indústria de equipamentos de transporte, alimentícia, borracha e plástico e a metal-mecânica. Também, ao contrário do resultado nacional, as indústrias listadas como heavy users apresentam um aumento médio de concentração significativo ao longo da década (Gráfico 1).

Por outro lado, no tocante ao nível de concentração espacial bruta da indústria, aquele que não leva em consideração ajustes derivados da microfundamentação do modelo EG, na média, observa-se uma certa desconcentração ao longo da década, exceto para as indústrias elétrica, alimentícia e automotiva (Gráfico 2). 
Tabela 3 - Índice de Herfindahl, conforme a metodologia proposta pelo Modelo EG (x 100)

\begin{tabular}{|c|c|c|c|c|c|c|c|c|c|c|c|c|}
\hline & 1999 & 2000 & 2001 & 2002 & 2003 & 2004 & 2005 & 2006 & 2007 & 2008 & 2009 & 2010 \\
\hline $\begin{array}{l}\text { FABRICAÇÃO DE OUTROS } \\
\text { EQUIPAMENTOS DE TRANSPORTE }\end{array}$ & 17,0 & 28,6 & 22,3 & 21,1 & 19,5 & 21,6 & 21,7 & 23,6 & 32,0 & 43,5 & 45,3 & 27,0 \\
\hline $\begin{array}{l}\text { FABRICAÇÃO DE MÁQUINAS, } \\
\text { APARELHOS E MATERIAIS ELÉTRICOS }\end{array}$ & 13,0 & 13,2 & 12,9 & 11,9 & 12,2 & 13,9 & 14,2 & 11,9 & 14,8 & 11,6 & 15,6 & 15,9 \\
\hline FABRICAÇÃO DE PRODUTOS DO FUMO & 12,5 & 16,4 & 14,3 & 13,0 & 14,8 & 17,7 & 23,7 & 11,2 & 11,5 & 17,9 & 12,3 & 14,4 \\
\hline $\begin{array}{l}\text { FABRICAÇÃO DE PRODUTOS DE } \\
\text { METAL - EXCLUSIVE MÁQUINAS } \\
\text { E EQUIPAMENTOS }\end{array}$ & 13,2 & 13,1 & 13,1 & 12,6 & 12,3 & 12,2 & 12,0 & 11,9 & 12,1 & 13,7 & 14,3 & 14,3 \\
\hline FABRICAÇÃO DE PRODUTOS QUÍMICOS & 3,5 & 3,5 & 3,6 & 3,6 & 3,3 & 3,1 & 3,0 & 3,4 & 3,5 & 3,4 & 4,2 & 3,9 \\
\hline $\begin{array}{l}\text { FABRICAÇÃO DE MÁQUINAS PARA } \\
\text { ESCRITÓRIO E EQUIPAMENTOS DE }\end{array}$ & 6,9 & 5,8 & 6,3 & 7,5 & 6,9 & 5,8 & 5,8 & 5,5 & 5,6 & 6,3 & 7,3 & 7,1 \\
\hline $\begin{array}{l}\text { EDIÇÃO, IMPRESSÃO E REPRODUÇÃO } \\
\text { DE GRAVAÇÕES }\end{array}$ & 1,5 & 1,6 & 1,6 & 1,4 & 1,5 & 1,4 & 1,2 & 1,2 & 1,1 & 1,0 & 1,7 & 1,7 \\
\hline $\begin{array}{l}\text { FABRICAÇÃO DE MATERIAL ELETRÔNICO } \\
\text { E DE APARELHOS E EQUIPAMENTOS }\end{array}$ & 3,9 & 3,6 & 3,9 & 3,8 & 3,7 & 3,7 & 4,2 & 3,6 & 4,7 & 3,9 & 3,9 & 4,0 \\
\hline FABRICAÇÃO DE PRODUTOS TÊXTEIS & 7,3 & 6,4 & 7,3 & 6,9 & 6,6 & 7,3 & 7,3 & 5,9 & 6,0 & 6,9 & 6,4 & 6,9 \\
\hline $\begin{array}{l}\text { FABRICAÇÃO DE CELULOSE, PAPEL E } \\
\text { PRODUTOS DE PAPEL }\end{array}$ & 7,6 & 7,5 & 6,7 & 9,5 & 10,1 & 10,4 & 10,5 & 7,5 & 8,3 & 7,4 & 6,5 & 6,5 \\
\hline $\begin{array}{l}\text { FABRICAÇÃO DE ARTIGOS DE BORRACHA } \\
\text { E PLÁSTICO }\end{array}$ & 17,0 & 16,4 & 17,4 & 16,6 & 17,6 & 18,1 & 18,3 & 17,4 & 17,2 & 16,8 & 16,4 & 15,9 \\
\hline $\begin{array}{l}\text { FABRICAÇÃO DE EQUIPAMENTOS DE } \\
\text { INSTRUMENTAÇÃO MÉDICO-HOSPITALAR }\end{array}$ & 16,6 & 18,6 & 17,4 & 16,8 & 16,6 & 15,7 & 15,9 & 18,5 & 16,8 & 16,9 & 15,5 & 15,2 \\
\hline $\begin{array}{l}\text { FABRICAÇÃO DE MÁQUINAS E } \\
\text { EQUIPAMENTOS }\end{array}$ & 7,3 & 8,2 & 7,9 & 7,9 & 8,4 & 6,8 & 7,2 & 5,8 & 5,3 & 5,5 & 5,5 & 5,9 \\
\hline $\begin{array}{l}\text { FABRICAÇÃO DE PRODUTOS } \\
\text { ALIMENTÍCIOS E BEBIDAS }\end{array}$ & 20,9 & 21,4 & 21,9 & 21,9 & 22,2 & 19,6 & 19,2 & 19,5 & 17,5 & 17,6 & 19,0 & 19,4 \\
\hline FABRICAÇÃO DE PRODUTOS DE MADEIRA & 3,7 & 3,7 & 4,4 & 4,0 & 3,0 & 2,6 & 2,5 & 1,9 & 2,1 & 2,1 & 2,0 & 2,2 \\
\hline $\begin{array}{l}\text { FABRICAÇÃO DE COQUE, REFINO DE } \\
\text { PETRÓLEO, ELABORAÇÃO DE COMBUST }\end{array}$ & 11,7 & 10,9 & 10,4 & 10,7 & 10,2 & 10,2 & 9,4 & 9,9 & 9,3 & 9,4 & 9,0 & 9,9 \\
\hline METALURGIA BÁSICA & 9,8 & 10,0 & 10,3 & 10,2 & 10,2 & 10,0 & 10,6 & 6,3 & 6,7 & 6,8 & 7,0 & 7,1 \\
\hline $\begin{array}{l}\text { CONFECÇÃO DE ARTIGOS DO } \\
\text { VESTUÁRIO E ACESSÓRIOS }\end{array}$ & 11,0 & 10,2 & 8,4 & 8,0 & 6,5 & 6,9 & 6,2 & 7,2 & 6,5 & 6,8 & 7,1 & 7,2 \\
\hline $\begin{array}{l}\text { PREPARAÇÃO DE COUROS E } \\
\text { FABRICAÇÃO DE ARTEFATOS } \\
\text { DE COURO }\end{array}$ & 9,9 & 7,1 & 8,0 & 7,4 & 7,8 & 6,1 & 6,1 & 5,1 & 5,1 & 3,9 & 4,1 & 4,0 \\
\hline $\begin{array}{l}\text { FABRICAÇÃO DE MÓVEIS E } \\
\text { INDÚSTRIAS DIVERSAS }\end{array}$ & 22,3 & 23,4 & 22,2 & 22,7 & 19,2 & 17,9 & 19,3 & 15,6 & 15,8 & 15,0 & 15,2 & 14,5 \\
\hline $\begin{array}{l}\text { FABRICAÇÃO DE PRODUTOS DE } \\
\text { MINERAIS NÃO-METÁLICOS }\end{array}$ & 24,9 & 25,2 & 24,7 & 26,2 & 24,3 & 23,1 & 21,6 & 12,8 & 17,4 & 14,0 & 14,7 & 12,4 \\
\hline $\begin{array}{l}\text { FABRICAÇÃO E MONTAGEM DE VEÍCULOS } \\
\text { AUTOMOTORES, } \\
\text { REBOQUES E CARROCERIA }\end{array}$ & 54,2 & 52,4 & 52,4 & 52,4 & 56,6 & 59,3 & 42,2 & 48,2 & 46,8 & 45,6 & 41,9 & 39,3 \\
\hline RECICLAGEM & 37,3 & 28,0 & 25,7 & 27,1 & 26,5 & 26,5 & 27,8 & 25,8 & 24,6 & 24,8 & 19,2 & 18,0 \\
\hline INDÚSTRIA TOTAL & 14,5 & 14,6 & 14,0 & 14,1 & 13,9 & 13,9 & 13,5 & 12,2 & 12,7 & 13,1 & 12,8 & 11,9 \\
\hline
\end{tabular}

Fonte: Elaboração própria. 
Por fim, o nível de concentração espacial, de acordo com a metodologia de Ellison e Glaeser (1994), vem se reduzindo em São Paulo. A indústria heavy user, embora mais concentrada que a média dos segmentos, também diminui seu nível de concentração espacial ao longo da década de 2000. O Gráfico 3 mostra que ao longo dos cinco últimos anos da década houve uma redução significativa da concentração para o grupo de consumidores intensivos de gás.

Quando se compara o conjunto de setores heavy users de gás natural com a indústria total, partindo do mesmo patamar de concentração espacial em 1999, o que se observa é uma progressiva redução, com os segmentos de consumo intensivo se desconcentrando mais rapidamente nos últimos cinco anos, de tal forma que se tornam menos concentrados que a média da indústria. O grupo de segmentos industriais potencialmente consumidores de gás natural, conforme definição da seção anterior, também apresenta uma desconcentração mais acelerada nos últimos anos.

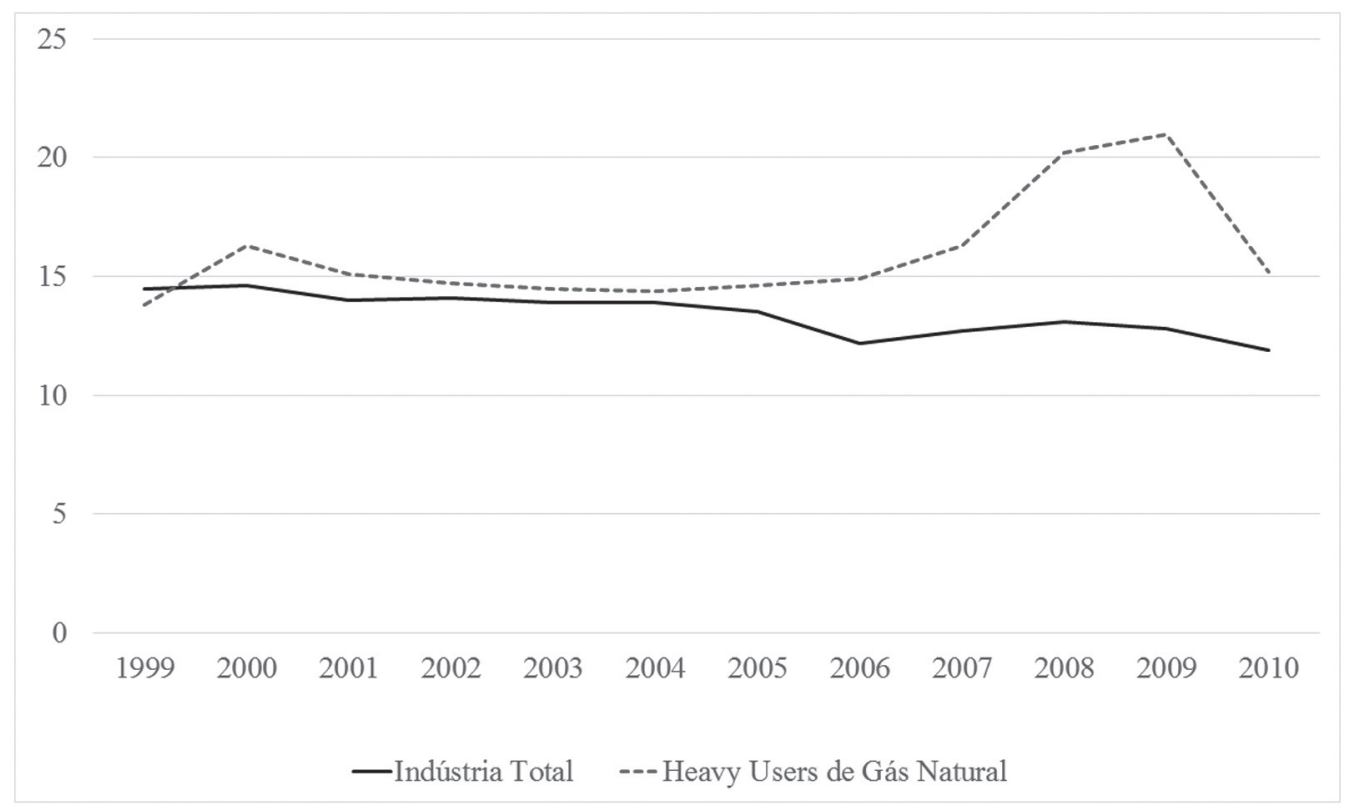

Gráfico l - Evolução do Índice de Herfindahl para o estado de São Paulo. 


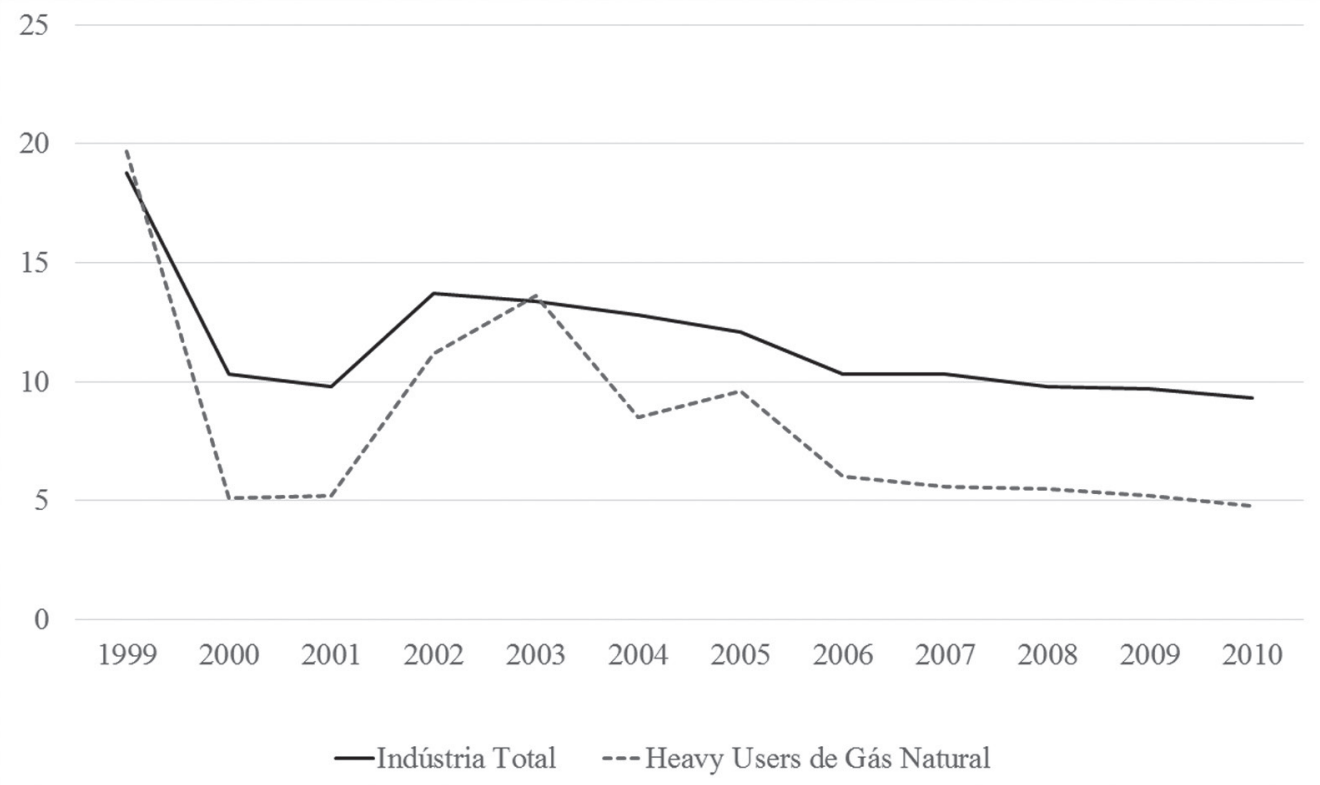

Fonte: Elaboração própria.

Gráfico 2 - Evolução da concentração geográfica bruta para o estado de São Paulo.

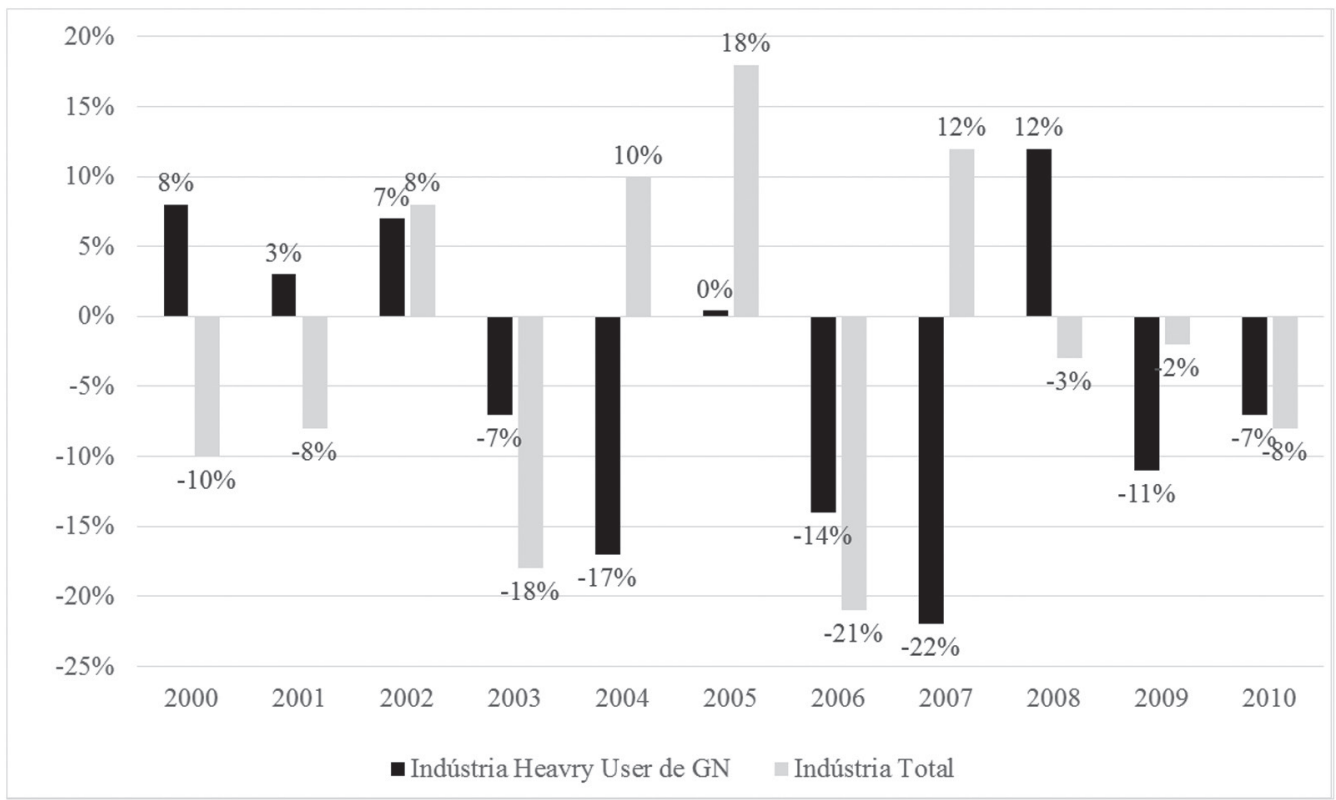

Fonte: Elaboração própria.

Gráfico 3 - Variações anuais do índice de concentração geográfica para o estado de São Paulo (\%). 


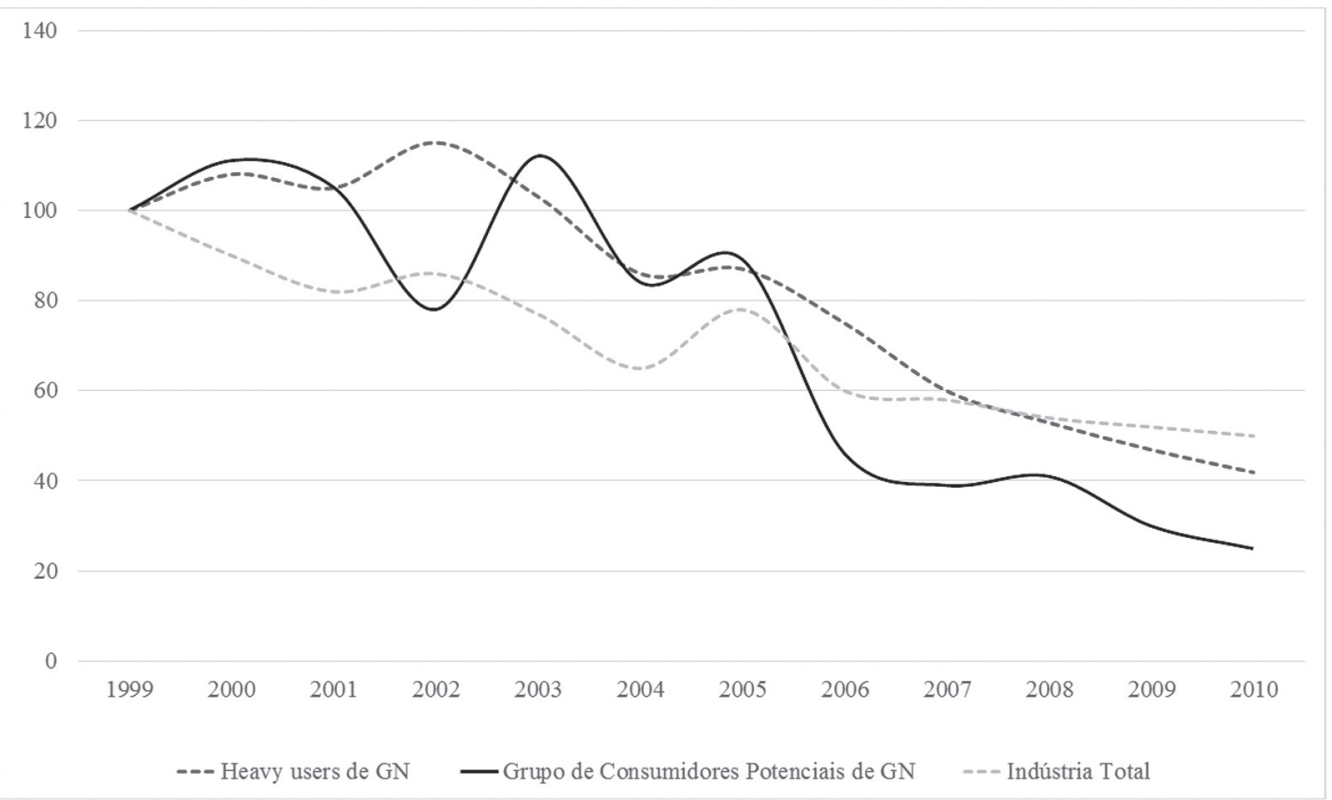

Fonte: Elaboração própria.

Gráfico 4 - Índice de Ellison e Glaeser para indústria total, segmentos heavy users de gás natural e segmentos potencialmente consumidores de gás natural em São Paulo (índice 1999=100).

Dessa maneira, não foi possível encontrar uma relação imediata entre o nível de concentração industrial e aqueles setores em que o consumo de gás natural na matriz energética tem grande peso relativo.

Para o estado de São Paulo, contudo, há um conjunto mais significativo de informações, permitindo que se desenvolvam análises mais amplas com a utilização das ferramentas espaciais. Mais do que calcular a formação de clusters industriais é possível desenvolver uma análise de correlação espacial entre a concentração industrial em um dado município e a disponibilidade de gás na região.

Os clusters industriais presentes no estado não se diferenciam significativamente ao longo do tempo. Também não se notam diferenças evidentes na formação de aglomerados quando se observa a indústria total ou os segmentos heavy users de gás natural. Os conjuntos de clusters estão basicamente concentrados nas regiões de polos industriais, como a Região Metropolitana de São Paulo, de Campinas e Vale do Paraíba. Como se verificou a existência de clusters industriais para o estado de São Paulo, é possível que esteja presente na decisão de localização da firma uma componente relacionada à economia de aglomeração. Ou seja, uma dada localidade pode se tornar mais atrativa quando já existe certo grau de especialização.

Há um maior conjunto de informações disponíveis para a distribuição de gás natural no estado de São Paulo. Portanto, foi possível aprofundar a análise da correlação espacial entre disponibilidade de gás e emprego industrial, através 
de um modelo econométrico simples que busca correlacionar a presença de gasodutos de distribuição de gás natural ${ }^{1}$ e o número de empregos na indústria.

Os resultados indicam que a presença de gasodutos de distribuição de gás tem uma correlação positiva e bastante significativa com o volume de emprego na região, mesmo quando há controle para custos de transporte e capital humano. No modelo que considera o emprego total, a dummy que controla a presença de gasodutos de distribuição se mostra insignificante, apesar de apresentar o sinal esperado correto. Os sinais das variáveis de controle se mostram consistentes com o esperado: a distância rodoviária em relação à capital apresenta sinal negativo, indicando que o aumento do custo de transporte inibe a localização industrial. Por outro lado, a presença de mão de obra qualificada atrai empresas industriais.

Em ambos os casos, a presença de gás natural leva a uma maior quantidade ou maior variação de emprego industrial ao longo do tempo. $\mathrm{O}$ fato de que a presença de gasodutos se mostra significativa apenas quando se considera o emprego industrial dos segmentos heavy users torna essa evidência ainda mais significativa. Pode-se assumir que os resultados para a indústria como um todo eliminam o efeito placebo e dão indício de que a variável dummy não está captando outros efeitos que não o esperado.

\section{Considerações finais}

A hipótese motivadora deste trabalho está relacionada ao potencial papel de indutor do desenvolvimento regional que a disponibilidade de energia possui. O desenvolvimento regional, nesse caso, é medido pela maior presença de atividade industrial.

Uma vez que a presença de gasodutos de transporte de gás natural exibe um evidente padrão de concentração espacial, seria natural esperar que as indústrias que levassem em conta a disponibilidade do energético em sua decisão de localização se tornassem mais concentradas, acompanhando o traçado dos principais gasodutos. Dessa maneira, a avaliação da hipótese poderia ser feita tanto por índices de concentração geográfica quanto por inspeções visuais das aglomerações industriais mapeadas.

Evidentemente, a disponibilidade de energia não é o único fator que leva à concentração industrial. Pode-se citar, por exemplo, a região onde o Gasbol está presente. Seu traçado coincide com uma das mais ricas regiões do país, onde existe um importante centro consumidor, além de estar próxima dos principais portos do Brasil. O mesmo pode ser dito do litoral, outra região que concentra a malha de gasodutos.

Os testes econométricos realizados para o estado de São Paulo se mostram mais robustos como indicativos do processo considerado. Uma vez que as variáveis concorrentes tenham seus efeitos isolados, é possível determinar de fato a importância da disponibilidade de gás natural sobre a decisão de localização. 
O que se verificou é que de fato a disponibilidade de gás natural teve importância na localização industrial. Tal resultado é corroborado pela avaliação de que seu efeito é mais significativo para a indústria consumidora intensiva de gás natural do que para a média das indústrias.

Por outro lado, há que considerar que esta análise também está limitada pelo conjunto de variáveis disponíveis para o controle. Embora tenham sido incluídas no modelo variáveis fundamentais no processo de localização, de acordo com as principais correntes teóricas, é inevitável que haja um grupo de variáveis omitidas. Cita-se, por exemplo, a relação de preços relativos entre os energéticos (gás natural em relação aos seus principais substitutos), as questões tributárias (guerra fiscal), a presença de centros econômicos regionais (os modelos consideraram apenas a distância em relação à capital, deixando de lado a existência de outros grandes centros consumidores), a proximidade com pontos de exportação e a proximidade com outras fontes de insumos importantes.

Essa constatação, por sua vez, não diminui a relevância dos testes produzidos. Ao contrário, se se pode provar que a malha de gasodutos do país serve como fator de atração de atividade econômica, e é quantitativamente significante neste processo, pode-se apontar mais uma possibilidade de atuação do setor público no sentido de garantir um processo de desconcentração econômica mais efetiva no país. A construção de uma malha mais eficiente e abrangente surge, portanto, como um importante desafio a ser considerado no planejamento energético nacional.

Nota

l A variável disponível para esta análise é a presença de city gates, ponto onde os gasodutos de transporte disponibilizam o gás natural para o sistema de distribuição, chegando ao consumidor final.

\section{Referências}

+GÁS BRASIL. Gás natural como fator de desenvolvimento. Disponível em: <http:// www.maisgasbrasil.com.br/>. Acesso em: 20 nov. 2012.

ABDEL-RAHMAN, H.; FUJITA, M. Product variety, Marshallian externalities and city sizes. Journal of Regional Science, v.30, n.2, p.165-83, 1990.

AGÊNCIA NACIONAL DO PETRÓLEO. Gás Natural e Biocombustíveis (ANP). Boletim Mensal do Gás Natural, n.29, 2011.

ALMEIDA, E. S.; HADDAD, E. A.; HEWINGS, G. J. D. The spatial analysis of crime in Minas Gerais: an exploratory analysis. São Paulo: Nereus, 2003. 
ANDRADE, T. A.; SERRA, R. V. Distribuição espacial do emprego e do produto industrial na década de 90: possibilidades atuais para a sua investigação. Estudos Econômicos, v.30, n.2, abr.-jun., 2000.

ANSELIN, L. Spatial econometrics. Boston: Kluwer Academic, 1988.

Local Indicators of Spatial Association - LISA. Geographical Analysis, v.27, n.2, p.93-115, 1996.

The Moran Scatterplot as an ESDA tool to assess Local Instability in Spatial Association. In: Spatial Analytical Perspectives on GIS in Environmental and Socio-Economic Sciences. London: Taylor and Francis, 1996. p.111-125.

BRASIL. Ministério do Planejamento, Orçamento e Gestão. Classificação Nacional de Atividades Econômicas 1.0. Disponível em: <http://www.cnae.ibge.gov.br/estrutura. asp>. Acesso em: $1^{\circ}$ fev. 2012.

CANO, W. Desequilibrios regionais e concentração industrial no Brasil: 1930-1995. Campinas: Unicamp, 1998.

CHAGAS, A. L. S.; TONETO JÚNIOR, R. Fatores determinantes do crescimento local: evidências a partir de dados dos municípios brasileiros para o período 1980-1991. IPEA, 2003.

CHAGAS, A. L. S. Externalidades de aglomeração: microfundamentação e evidências empíricas. São Paulo, 2004. Dissertação (Mestrado) - Universidade de São Paulo. São Paulo, 2004.

DEVEREUX, M. P.; GRIFFITH, R.; SIMPSON, H. The geographic distribution of production activity in the UK. Regional Science and Urban Economics, n.34, p.533-64, 2004.

DINIZ, C. C. A dinâmica regional recente da economia brasileira e suas perspectivas. IPEA, Texto para Discussão, n.375, jun. 1995.

DOMINGUES, E. P. Aglomerações e periferias industriais no Brasil e no Nordeste. Revista Econômica do Nordeste, v.36, n.4, p.508-23, 2005.

EDWARDS, M. E. Regional and urban economics and economic development: Theory and methods. s. 1.: Auerbach Publications, 2006.

ELLISON, G.; GLAESER, E. L. Geographic concentration in U.S. Manufacturing industries: a dartboard approach. NBER Working Paper, n.4840, 1994.

EMPRESA DE PESQUISA ENERGÉTICA. Balanço Energético Nacional-BEN 2012. 282 p. Rio de Janeiro, 2012a.

EMPRESA DE PESQUISA ENERGÉTICA. Plano Decenal de Expansão de Energia 2021 - PDE 2021. Rio de Janeiro, 2012b.

FARIA, L. F. R. A integração dos mercados de gás natural e energia elétrica no Brasil. Belo Horizonte, 2010. Dissertação (Mestrado) - Universidade Federal de Minas Gerais. Belo Horizonte, 2010.

FUJITA, M.; THISSE, J. F. Economics of agglomeration: cities, industrial location and regional growth. Cambridge: Cambridge University Press, 2001.

GASNET. Mapas de Gasodutos - Gasodutos em Operação. Disponível em: <http://www. gasnet.com.br/novo_gasoduto/operacao.asp>. Acesso em: 10 nov. 2012.

GLAESER, E.; SCHEINKMAN, J.; SHLEIFER, A. Economic growth in a cross-section of cities. Cambridge, Mass: NBER, 1995. 
HIRSCHMAN, A. O. The paternity of an index. The American Economic Review, v.54, n.5, p.761, set. 1964 .

IBGE. Estados@. Disponível em: <http://ibge.gov.br/estadosat/>. Acesso em: 15 out. 2012.

KRUGMAN, P. Increasing returns and economic geography. Journal of Political Economy, v.99, n.3, p.483-99, 1991.

LAUTERT, V.; ARAÚJO, N. C. M. Concentração industrial no Brasil no período 1996-2001: uma análise por meio do índice de Ellison e Glaeser (1994). Economia Aplicada, v.11, n.3, p.347-68, jul./set. 2007.

MARQUES, F. M. R. Redução de assimetria de informação na revisão tarifária da distribuição de gás canalizado no Brasil: proposta de uma metodologia baseada na análise de geração de valor. São Paulo, 2009. Tese (Doutorado) - Universidade de São Paulo. São Paulo, 2009.

MARSHALL, A. Principles of economics. s. 1.: McMillan, 1920.

MAUREL, F.; SÉDILLOT, B. A measure of the geographic concentration in French manufacturing industries. Regional Science and Urban Economics, n.29, p.575-604, 1999.

McCANN, P. Industrial Location Economics. Cheltenhan: Edward Elgan, 2002.

MENEZES, M. G. C. A cidade em movimento: a distribuição e o crescimento da atividade econômica no espaço urbano brasileiro no período de 1970 a 2002. Belo Horizonte, 2006. Dissertação (Mestrado) - Universidade Federal de Minas Gerais. Belo Horizonte, 2006.

MOUTINHO DOS SANTOS, E. et al. Gás natural: estratégias para uma energia nova no Brasil. São Paulo: Annablume, 2002.

NIJKAMP, P.; PERRELS, A. Impacts of electricity rates on industrial location. Energy Economics, 1988.

OLIVEIRA, C. A. Crescimento econômico das cidades nordestinas: um enfoque da novas geografia econômica. In: IX ENCONTRO REGIONAL DE ECONOMIA, 2004. Anais..., 2004.

PACHECO, C. A. Novos padrões de localização industrial? Tendências recentes dos indicadores de produção e do investimento industrial. Textos para Discussão IPEA, n.633, 1999.

PIQUET, R.; MIRANDA, E. A indústria de gás no Brasil: incertezas, implicações territoriais e perspectivas. Novos Cadernos NAEA, v.12, n.1, p.51-66, 2009.

RESENDE, M.; WYLLIE, R. Aglomeração industrial no Brasil: um estudo empírico. Estudos Econômicos, v.35, n.3. p.433-60, jul./set. 2005.

RIGOLIN, P. H. C. Avaliação global dos modos energéticos de transporte do gás natural inclusive como energia secundária. São Paulo, 2007. 101f. Dissertação (Mestrado) Universidade de São Paulo. Faculdade de Economia e Administração, São Paulo, 2007.

SANTOS, G. F. Politica energética e desigualdades regionais na economia brasileira. São Paulo, 2012. Tese (Doutorado) - Faculdade de Economia e Administração. Universidade de São Paulo, São Paulo, 2012.

SILVA, M. V. B.; SILVEIRA NETO, R. M. Dinâmica da concentração da atividade in- 
dustrial no Brasil entre 1994 e 2004: uma análise a partir de economias de aglomeração e da nova geografia econômica. Economia Aplicada, v.13, n.2, p.299-331, 2009.

SILVEIRA NETO, R. M. Concentração industrial regional, especialização geográfica e geografia econômica: evidências para o Brasil no período 1950-2000. Revista Econômica do Nordeste, v.36, n.3, p.189-208, 2005.

SUZIGAN, W. et al. Aglomerações industriais no Estado de São Paulo. Economia Aplicada, v.5, n.4, p.695-717, out./dez. 2001.

TIROLE, J. The theory of industrial organization. Cambridge: The MIT Press, 1988.

TRANSPORTADORA BRASILEIRA GASODUTO BOLÍVIA BRASIL (TGB). Mapa de Gasodutos. Disponível em: <http://www.tbg.com.br/portalTBGWeb/tbg.portal?_ nfpb=true\&_pageLabel=pgTracadoGasoduto $>$. Acesso em: 10 nov. 2012.

WEBER, A. Theory of the location of industries. Chicago: The University of Chicago Press, 1929.

WORREL, E. et al. Energy intensity in the iron and steel industry: a comparison of physical and economic indicators. Energy Policy, v.25, n.7-9, p.727-44, 1997.

RESUMO - A utilização de gás natural tem ganhado grande espaço na matriz energética brasileira, principalmente dentro do setor industrial. Para um conjunto de segmentos e processos industriais, a utilização de gás natural como energético ou matéria-prima envolve significativos ganhos dos pontos de vista ambiental, técnico e econômico. A hipótese motivadora deste trabalho está relacionada ao potencial papel de indutor do desenvolvimento regional que a disponibilidade de energia possui. Foram definidas duas principais vias de análise: (i) a avaliação do nível de concentração geográfica (ou espacial) da indústria, verificando-se a existência de padrões distintos para a média da indústria e um grupo de setores definidos como heavy users de gás natural (cerâmica, têxtil e química), e (ii) a avaliação e mapeamento das correlações espaciais entre o emprego industrial (proxy da atividade industrial) e a presença de gasodutos. Para o estado de São Paulo verificou-se que existe uma forte correlação positiva entre a presença de gasodutos de distribuição de gás natural e o nível de emprego para os segmentos heavy users, mesmo quando se consideram os efeitos de outras variáveis relevantes, tais como custos de transporte, qualificação da mão de obra e ganhos com a especialização.

PALAVRAS CHAVES: Gás natural, Localização industrial, Gasodutos, Análise espacial.

ABSTRACT - The use of natural gas has gained ground in the Brazilian energy matrix, especially in the industrial sector. For various segments and industrial processes, the use of natural gas as fuel or raw material represents significant environmental, technical and economic gains. The hypothesis motivating this paper is the potential role of the availability of energy as a promoter of regional development. We defined two main pathways of analysis: (i) assessment of the level of geographical (or spatial) concentration of the industry, whereby we ascertained the existence of distinct patterns for the industry as a whole, as well as a group of sectors defined as heavy users of natural gas (ceramics, textiles and chemicals); and (ii) assessment and mapping of the spatial correlations between 
employment in manufacturing (a proxy for industrial activity) and the presence of gas pipelines. We found that in the state of São Paulo there is a strong positive correlation between the presence of gas pipelines and the employment level among heavy users, even taking into account the effects of other relevant variables, such as transportation costs, qualification of labor and gains from specialization.

KEYWORDS: Natural gas, Industrial location, Gas pipelines, Spatial analysis.

Edgar Antonio Perlotti é economista, mestre em Ciências pelo Programa de Pós-Gra-duação em Energia da Universidade de São Paulo (PPGE/USP).

@ - edperlotti@gmail.com

Edmilson Moutinho dos Santos é doutor, professor do Programa de Pós-Graduação em Energia da Universidade de São Paulo (PPGE/USP) e coordenador do Programa de Políticas de Energia e Economia do Centro de Pesquisa para Inovação em Gás.

@ - edsantos@iee.usp.br

Hirdan Katarina de Medeiros Costa é advogada, doutora em Ciências pelo Programa de Pós-Graduação em Energia da Universidade de São Paulo (PPGE/USP) e vice coordenadora do Programa de Políticas de Energia e Economia do Centro de Pesquisa para Inovação em Gás. @ - hirdan@usp.br ; hirdankatarina@gmail.com

Recebido em 28.11.2013 e aceito em 2.7.2015.

I, II, III Programa de Pós-Graduação em Energia, Universidade de São Paulo, São Paulo/ São Paulo, Brasil.

Agradecimentos - Os autores agradecem o suporte da BG E\&P Brasil e Fapesp por meio do Centro de Pesquisa e Inovação do Gás (Fapesp Proc. 2014/50279-4), assim como a da Universidade de São Paulo e a importância estratégica do suporte dado pela ANP (Agência Nacional de Petróleo, Gás Natural e Biocombustíveis) por meio da cláusula de P\&D e do apoio do Programa de Recursos Humanos (PRH) 04/ANP/MCTI. 